\title{
Smoking and impact on health
}

\author{
P. Vineis
}

ABSTRACT: In 2005, the World Health Organization set a global goal to reduce the rate of death from chronic (noncommunicable) disease by an additional $2 \%$ every year. A major component in this strategy was a reduction in the use of tobacco products, as described in the World Health Organization Framework Convention on Tobacco Control. According to recent estimates, over 10 yrs (2006-2015) 13.8 million deaths could be averted by the implementation of such interventions, at a cost of less than US\$0.40 person ${ }^{-1} \cdot \mathrm{yr}^{-1}$ in low-income and lower middleincome countries, and US\$0.50-1.00 person $^{-1} \cdot \mathrm{yr}^{-1}$ in upper middle-income countries. According to estimates, approximately one third of tobacco-related deaths will be due to respiratory causes, one third to cancer and one third to cardiovascular diseases. Most of the burden of tobacco in the future will be in low-income countries.

KEYWORDS: Health problems, smoking, tobacco

I $\mathrm{t}$ has been repeatedly stated by international agencies that tobacco is the main cause of death in most countries. The extent of diseases and disabilities related to tobacco has not been completely explored, since only some categories of disease (cancer, cardiovascular, respiratory) have been extensively and accurately investigated. If current smoking patterns continue, there will be more than one billion deaths attributable to tobacco smoking in the 21st Century compared with $\sim 100$ million deaths in the 20th Century. The only other causes of disease with such rapidly increasing impact are those associated with HIV infection and, perhaps, obesity in Western countries.

The present article will examine some of the health effects of tobacco smoking, mainly in relation to cancer and cardiovascular diseases, and then examine the total burden of disease attributable to tobacco worldwide.

\section{CARCINOGENIC EFFECTS}

According to a recent systematic evaluation, "...tobacco is a potent multisite carcinogen with a worldwide impact, causing cancers of the lung, upper aero-digestive tract (oral cavity, nasal cavity, nasal sinuses, pharynx, larynx, oesophagus), pancreas, stomach, liver, lower urinary tract (renal pelvis and bladder), kidney, uterine cervix and myeloid leukemia" [1]. Figure 1 shows the increase in mortality from lung cancer that occurred in the USA between 1930 and 2000. Similar trends have been described in Western countries and are now being described in many developing countries.

Table 1 summarises the evidence for the cancer sites for which a Working Group of the
International Agency for Research on Cancer (IARC) found "sufficient evidence" of carcinogenicity of tobacco smoke [1-3]. Overall tobacco accounts for $\sim 90 \%$ of all lung cancers in Western countries, and approximately one third of all deaths from cancer.

The causal nature of the association with the cancer sites indicated in table 1 is beyond any reasonable doubt, and is reinforced by two areas of evidence. First, smoking cessation is followed by a "freezing of the risk". As shown in figure 2, individuals who started smoking at 20 yrs of age have a cumulative lifetime risk of lung cancer of $\sim 15 \%$; however, if they quit by 50 yrs of age this risk is reduced to $6 \%$ and further reduced to $2 \%$ if they quit at 30 yrs of age, whereas never-smokers have a risk of $1 \%$ [4]. The second area of evidence is taken from mechanistic data, exemplified for lung cancer in figure 2.

Several types of mechanistic evidence complement the epidemiological findings. Overall, the mechanistic evidence concerning the measurement of metabolites of tobacco compounds, the formation of DNA or protein adducts, and the spectrum of gene mutations substantiates and elucidates the genetic and molecular changes induced by exposure to tobacco smoke, thus, addressing earlier criticisms pertaining to the limited understanding of the mechanisms of tobacco carcinogenicity. In the case of lung cancer, polycyclic aromatic hydrocarbons (PAH), carcinogenic compounds present in tobacco smoke, induce mutations in the p53 gene, which are crucial for cell-cycle deregulation and carcinogenesis. $\mathrm{G}$ to $\mathrm{T}$ transversions within the p53 gene have been linked to a molecular
CORRESPONDENCE

P. Vineis

Imperial College London

St Mary's Campus

Norfolk Place

London

W2 $1 P G$

UK

Fax: 442074022150

E-mail: p.vineis@imperial.ac.uk

STATEMENT OF INTEREST

None declared. 


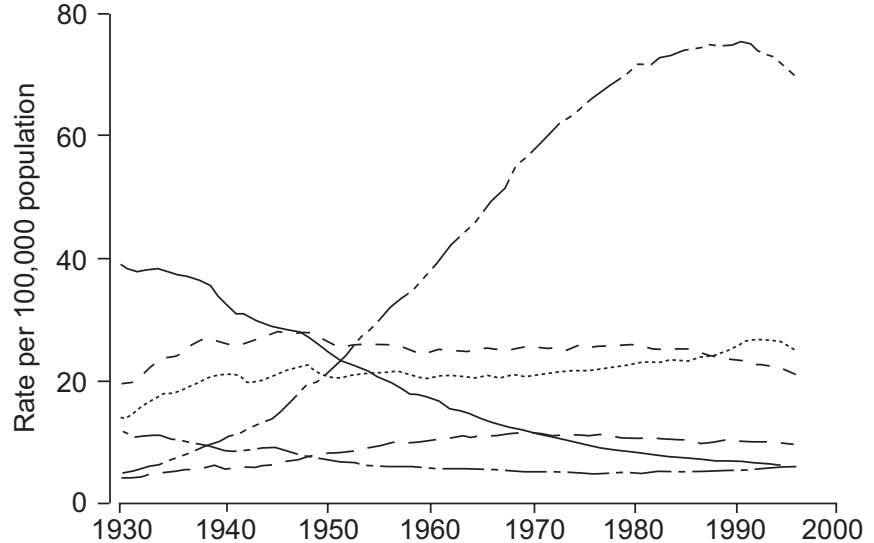

FIGURE 1. Trends of mortality rates in the USA between 1930 and 2000 in males according to cancer site. —_: stomach; ….... prostate; - — - : colon

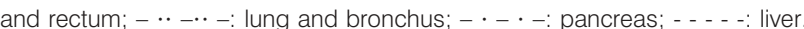

signature of tobacco mutagens in smoking-associated lung cancers for the following reasons. 1) PAHs are a major class of carcinogens in tobacco smoke that predominantly produce $G$ to $\mathrm{T}$ transversions (fig. 3). 2) PAH adducts are present in DNA extracted from human tissues exposed to tobacco smoke. 3) The frequency of $G$ to $T$ transversions in lung cancers from smokers is increased relative to the frequency in lung cancers from nonsmokers. 4) A nontranscribed strand bias of $\mathrm{G}$ to $\mathrm{T}$ transversions can be attributed to the preferential repair of adducts on the transcribed strand [5].

$\mathrm{N}$-nitroso compounds are another major group of chemicals found in tobacco smoke, several of which are potent animal carcinogens. $N$-nitroso compounds are found in the urine of smokers. In particular, compounds known as 4(methylnitrosamino)-1-(3-pyridyl)-1-butanol (NNAL) and NNAL-glucuronide are very useful biomarkers because they are derived from the carcinogen 4-(methylnitrosamino)-1-(3pyridyl)-1-butanone, which is specific to tobacco products [6] Cotinine is probably the best marker of exposure to tobacco smoke, but it is not directly relevant to carcinogenesis.

\section{ENVIRONMENTAL TOBACCO SMOKE}

Nonsmokers who breathe other people's smoke (i.e. involuntary smoking) inhale the same carcinogens as active smokers, although at much lower doses. Because smoking is an established cause of lung cancer in smokers, it follows that there must also be some risk of lung cancer to lifelong nonsmokers exposed to involuntary smoking. It is also likely that there will be some additional risk deriving from involuntary smoking in individuals who are now nonsmokers but who used to be smokers, compared with ex-smokers not exposed to involuntary smoking.

Most of the studies evaluating involuntary smoking (or environmental tobacco smoke) and risk of lung cancer in never-smokers compared risks for spouses of smokers with risks for spouses of nonsmokers. These studies have been carried out in several countries and, in general, indicate an increased risk, especially for those with high exposure. To evaluate the information collectively, in particular from those studies with a limited number of case subjects, meta-analyses

\begin{tabular}{|c|c|c|c|c|}
\hline \multirow{3}{*}{$\begin{array}{l}\text { TABLE } 1 \\
\text { Cancer site }\end{array}$} & \multicolumn{4}{|c|}{$\begin{array}{l}\text { Cancer sites for which there is "sufficient" } \\
\text { evidence of carcinogenicity of tobacco smoking } \\
\text { according to the International Agency for } \\
\text { Research on Cancer Working Group }\end{array}$} \\
\hline & & \multicolumn{2}{|c|}{ Studies } & \multirow[t]{2}{*}{ RR } \\
\hline & & Case-control & Cohort & \\
\hline Lung & & $>100$ & 37 & $15-30$ \\
\hline Urinary tract & & 50 & 24 & 3 \\
\hline \multicolumn{4}{|c|}{ Upper aero-digestive tract } & \\
\hline Oro-and hy & opharynx & 28 & 6 & $4-5$ \\
\hline Oesophage & & 45 & 19 & $1.5-5$ \\
\hline Larynx & & 25 & 5 & 10 \\
\hline Pancreas & & 38 & 27 & $2-4$ \\
\hline $\begin{array}{c}\text { Nasal cavity } \\
\text { sinuses }\end{array}$ & Jaranasal & 9 & 1 & $1.5-2.5$ \\
\hline Nasopharyn & & 9 & 2 & $1.5-2.5$ \\
\hline Stomach & & 44 & 27 & $1.5-2$ \\
\hline Liver & & 29 & 29 & $1.5-2.5$ \\
\hline Kidney & & 13 & 8 & $1.5-2.0$ \\
\hline Uterine cerv & & 49 & 14 & $1.5-2.5$ \\
\hline Myeloid leuk & emia & NR & 12 & $1.5-2$ \\
\hline
\end{tabular}

Data are presented as $\mathrm{n}$, unless otherwise stated. RR: relative risk; NR: no reviewed. Reproduced from [1, 2] with permission from the publisher

have been conducted in which the relative risk estimates from the individual studies were pooled. Nonsmokers have a statistically significant greater risk of lung cancer if their spouses are smokers than if their spouses are nonsmokers. The increase in risk remains after controlling for bias and potential confounding. From a recent meta-analysis performed by the IARC $[1,7]$, the risk was $\sim 25 \%$ greater than expected for females (based on data from 46 studies that included 6,257 lung cancer case-subjects) and 35\% greater than expected for

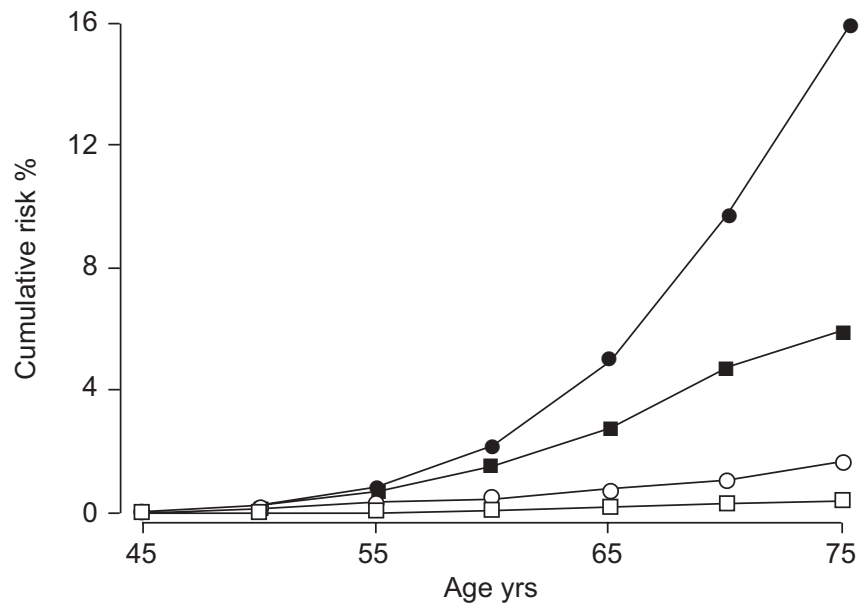

FIGURE 2. Cumulative risk of death from lung cancer current smokers; 口 : stopped smoking at age $50 \mathrm{yrs}$; $\bigcirc$ : stopped smoking at age $30 \mathrm{yrs}$; $\square$ : neversmokers. Adapted from [4] with permission from the publisher 

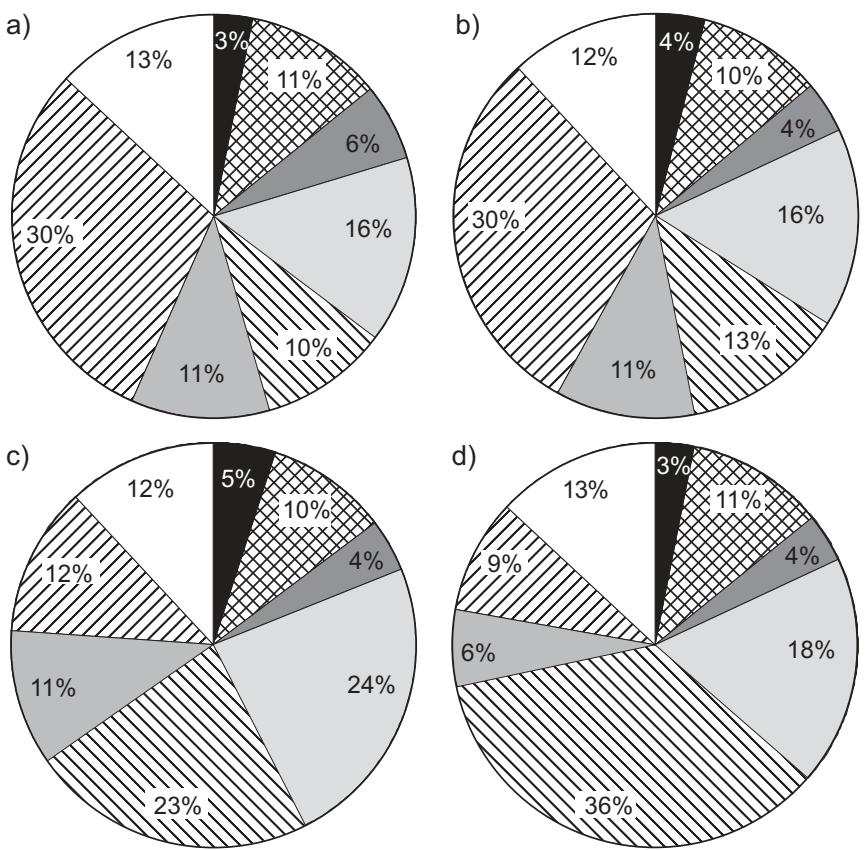

d)

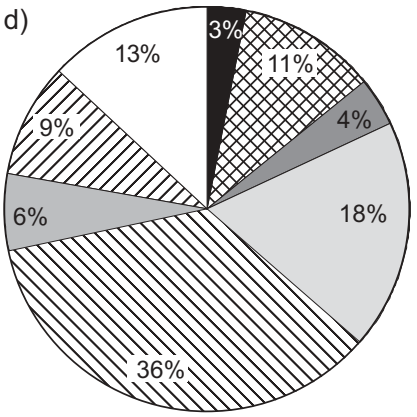

FIGURE 3. Patterns of p53 mutations according to smoking habits in a) all lung cancers minus nonsmokers $(n=1,289), b)$ smokers with lung cancer $(n=419)$, c) nonsmokers with lung cancer (153), and d) brain, breast and colorectal cancer

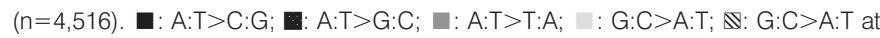
CpG; $\square: G: C>C: G ; \mathbb{Z}: G: C>T: A ; \square:$ deletion/insertion/complex. Reproduced from [5] with permission from the publisher.

males (based on data from 11 studies that included 442 lung cancer case-subjects). In addition, several studies have evaluated the risk of lung cancer among nonsmokers exposed to involuntary smoking at the workplace [7]. A meta-analysis by the IARC, based on 19 studies of females who did not smoke (including 3,588 lung cancer case-subjects), showed that the risk of lung cancer was $\sim 20 \%$ greater than expected. Of the studies examined by IARC [1], four had an odds ratio (OR) or relative risk $<1.0,19$ had an OR between 1.1 and 2.0, and 10 studies had an OR $>2$ (suggesting at least a doubling of the risk). This unbalanced distribution cannot be attributed to chance. Both case-control and cohort studies reported positive findings. Publication bias, which is a more frequent publication of positive findings than negative findings, has been ruled out in the meta-analysis. In fact, 300 unpublished studies would be needed to explain the overall OR that has been found, a clearly implausible assumption.

The biological plausibility of the association between the risk of lung cancer and involuntary smoking is supported by the fact that the urine of nonsmokers exposed to involuntary smoking contains concentrations of carcinogenic $N$-nitroso compounds specific to tobacco, which are between $1-5 \%$ of the concentrations found in the urine of active smokers, i.e. approximately proportional to the increased risk found in epidemiological studies of involuntary smoking. It is interesting to note that among the studies conducted to investigate the effects of environmental tobacco smoke, the studies that recorded negative findings were more frequently sponsored by the tobacco industry (table 2) [8].

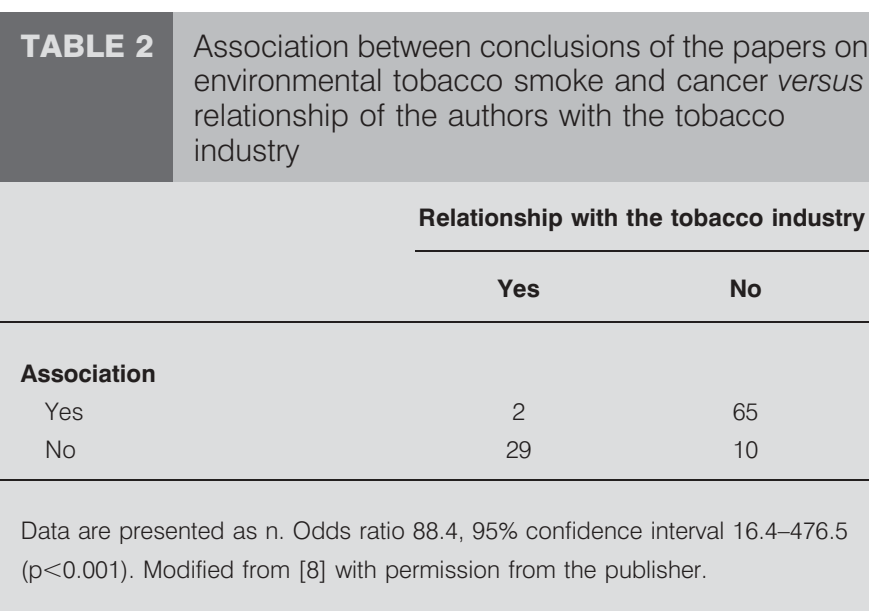

\section{CARDIOVASCULAR EFFECTS}

Smoking causes even more deaths from vascular, respiratory and other diseases than from cancer; therefore, in total, tobacco smoking is estimated to account for approximately four to five million deaths a year worldwide.

Table 3 shows the results of a recent study that examined cardiovascular diseases in relation to smoking. The effect of smoking on these outcomes, i.e. an approximate doubling of the risk of myocardial infarction and stroke, was the same among males with low cholesterol levels as among those with higher levels [9]. Smoking accounted for $20 \%$ of all cardiovascular disease outcomes in this population.

Animal research suggests that tobacco-smoke carcinogens can induce and stimulate a proliferative vascular smooth muscle cell phenotype and elevated DNA adducts have been found in the vascular tissue of smokers $[10,11]$.

\section{GLOBAL BURDEN}

In 2005, the World Health Organization (WHO) set a global goal to reduce the rate of death from chronic (noncommunicable) disease by an additional $2 \%$ every year. To this end, ASARIA et al. [12] investigated how many deaths could potentially be averted over $10 \mathrm{yrs}$ by the implementation of selected population-based interventions, and calculated the financial costs of their implementation. AsARIA et al. [12] selected two interventions: 1) to reduce salt intake in the population by $15 \%$; and 2 ) to implement four key elements of

TABLE 3 Prospective cohort study of 648,346 Korean males aged 30-64 yrs at their baseline assessment in 1992

\section{HR (95 Cl)}

Ischaemic stroke

Subarachnoid haemorrhage

Myocardial infarction

Aortic aneurysm
$1.58(1.49-1.68)$

$1.91(1.63-2.24)$

$2.01(1.87-2.17)$

$1.47(1.14-1.90)$
HR: hazard ratio; Cl: confidence interval. Modified from [9] with permission from the publisher. 

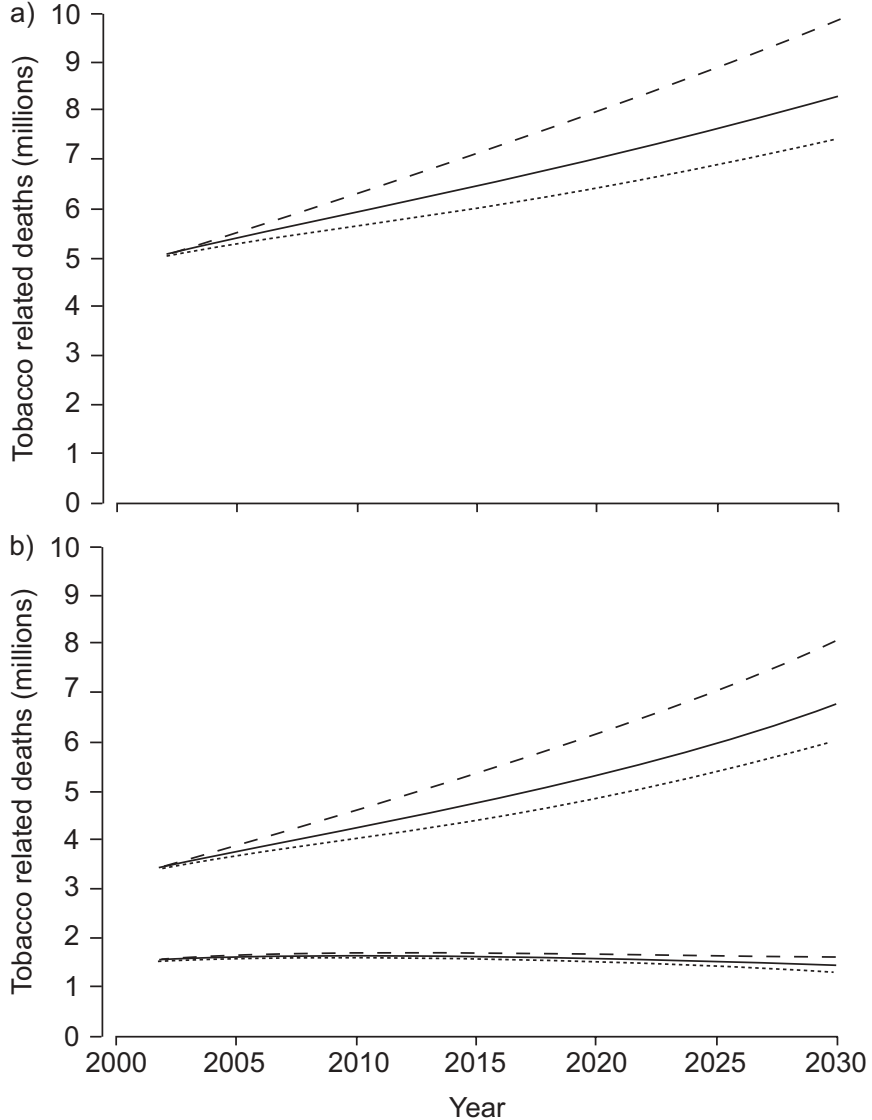

FIGURE 4. The projected numbers of tobacco-related deaths between 20002035 in millions for a) the world and b) medium- and low-income countries (top) and high-income countries (bottom). _- baseline; - - -: pessimistic; ........ optimistic. Reproduced from [13] with permission from the publisher.

the WHO Framework Convention on Tobacco Control. Methods from the WHO Comparative Risk Assessment project were used to: 1) estimate shifts in the distribution of risk factors associated with salt intake and tobacco use; and 2) model the effects on chronic disease mortality for 23 countries, which account for $80 \%$ of chronic disease burden in the developing world. AsARIA et al. [12] showed that, over 10 yrs (from 2006 to 2015), 13.8 million deaths could be averted by implementing these interventions at a cost of less than US $\$ 0.40$ person $^{-1} \cdot \mathrm{yr}^{-1}$ in low-income and lower middle-income countries, and US\$0.50-1.00 person ${ }^{-1} \cdot \mathrm{yr}^{-1}$ in upper middleincome countries (as of 2005). These two population-based intervention strategies could, therefore, substantially reduce mortality from chronic diseases and make a major (and affordable) contribution towards achieving the global goal to prevent and control chronic diseases [12].

MATHERS and LONCAR [13] have performed a similar study, again subdividing the projected numbers of tobacco-related deaths for high-income and middle- plus low-income countries using three scenarios relating to the period 2002-2030 (fig. 4). The worst-case scenario is one in which the current trends in tobacco sales continue to rise in low-income countries, the second (baseline) scenario is based on a moderate success of preventive measures, and the third scenario is an optimistic

\begin{tabular}{lcc} 
TABLE 4 & $\begin{array}{c}\text { Projected global tobacco-related deaths, by } \\
\text { cause, 2015: baseline scenario }\end{array}$ \\
\cline { 2 - 3 } Cause & \multicolumn{2}{c}{ Tobacco-related deaths } \\
\cline { 2 - 3 } & N millions & Total \% \\
\hline All causes & 6.43 & 100 \\
Tuberculosis & 0.09 & 1 \\
Lower respiratory infections & 0.15 & 2 \\
Malignant neoplasms & 2.12 & 33 \\
Trachea, bronchus, lung cancers & 1.18 & 18 \\
Mouth and oropharynx cancers & 0.18 & 3 \\
Oesophagus cancer & 0.17 & 3 \\
Stomach cancer & 0.12 & 2 \\
Liver cancer & 0.10 & 2 \\
Other malignant neoplasms & 0.34 & 5 \\
Diabetes mellitus & 0.13 & 2 \\
Cardiovascular diseases & 1.86 & 29 \\
Ischaemic heart disease & 0.93 & 14 \\
Cerebrovascular disease & 0.52 & 8 \\
Other cardiovascular diseases & 0.24 & 4 \\
Respiratory diseases & 1.87 & 29 \\
COPD & 1.76 & 3 \\
Digestive diseases & 0.20 & \\
\hline & &
\end{tabular}

COPD: chronic obstructive pulmonary disease. Reproduced from [13] with permission from the publisher

one in which preventive campaigns are more successful. It is clear that most, if not all, of the burden of tobacco in the future will be in low-income countries. Finally, table 4 contains estimates of the millions of deaths attributable to smoking in the world according to the baseline scenario, projected to 2015 and divided by specific causes. Approximately one third of tobacco-related deaths will be due to respiratory causes, one third to cancer and one third to cardiovascular diseases.

\section{REFERENCES}

1 World Health Organization, International Agency for Research on Cancer. IARC Monographs on the Evaluation of Carcinogenic Risks to Humans. Tobacco Smoking and Involuntary Smoking. Vol. 83. Lyon, IARC, 2004.

2 Vineis P, Alavanja M, Buffler P, et al. Tobacco and cancer: recent epidemiological evidence. J Natl Cancer Inst 2004; 96: 99-106.

3 Doll R. Uncovering the effects of smoking: historical perspective. Stat Methods Med Res 1998; 7: 87-117.

4 Peto R, Darby S, Deo H, Silcocks P, Whitley E, Doll R. Smoking, smoking cessation, and lung cancer in the UK since 1950: combination of national statistics with two casecontrol studies. BMJ 2000; 321: 323-329.

5 Hainaut P, Pfeifer GP. Patterns of p53 G to T transversions in lung cancers reflect the primary mutagenic signature of DNA-damage by tobacco smoke. Carcinogenesis 2001; 22: 367-374.

6 Hecht SS. Human urinary carcinogen metabolites: biomarkers for investigating tobacco and cancer. Carcinogenesis 2002; 23: 907-922. 
7 Hackshaw AK, Law MR, Wald NJ. The accumulated evidence on lung cancer and environmental tobacco smoke. BMJ 1997; 315: 980-988.

8 Barnes DE, Bero LA. Why review articles on the health effects of passive smoking reach different conclusions. JAMA 1998; 279: 1566-1570.

9 Lawlor DA, Song YM, Sung J, Ebrahim S, Smith GD. The association of smoking and cardiovascular disease in a population with low cholesterol levels: a study of 648,346 men from the Korean national health system prospective cohort study. Stroke 2008; 39: 760-767.

10 Van Schooten FJ, Hirvonen A, Maas LM, et al. Putative susceptibility markers of coronary artery disease: association between VDR genotype, smoking, and aromatic DNA adduct levels in human right atrial tissue. FASEB J 1998; 12: 1409-1417.

$11 \mathrm{Li} J M$, Cui TX, Shiuchi $\mathrm{T}$, et al. Nicotine enhances angiotensin II-induced mitogenic response in vascular smooth muscle cells and fibroblasts. Arterioscler Thromb Vasc Biol 2004; 24: 80-84.

12 Asaria P, Chisholm D, Mathers C, Ezzati M, Beaglehole R. Chronic disease prevention: health effects and financial costs of strategies to reduce salt intake and control tobacco use. Lancet 2007; 370: 2044-2053.

13 Mathers CD, Loncar D. Projections of global mortality and burden of disease from 2002 to 2030. PLoS Med 2006; 3: e442. 